\title{
Epidermal Flexible Printable Non Contact Autonomous ECG Sensor Medical Device Patch for Early Warning
}

\author{
Er M Sesha Giri Rao ${ }^{1}$, Dr V S Chouhan ${ }^{2}$ \\ ${ }^{I}$ (Department of Electronics and Information Technology, Government of India ) \\ ${ }^{2}$ (Faculty, MBM Engineering College, Jodhpur)
}

\begin{abstract}
Flexible biosensor based wearable medical devices can act as excellent terminals in the body area electronics of health monitoring system. Benefiting from the growing advancement of electronics and communication technique are wearable medical devices, which can be worn by people and measure physiological parameters like Electro Cardio Gram (ECG), make it possible to monitor the health status continuously to observe deterioration if any, on-line basis, without causing any discomfort to the subjects or patients, in their daily life and could provide warning, even during sleep and wake up the Cardio Vascular or sleep apnea patients. Among all the deaths caused by Cardio Vascular Diseases (CVDs) about two-thirds of them happen in Out-side hospital environment. There is a need to prevent the development of CVDs at its early stage, so as to provide a low cost and efficient way to realize pervasive healthcare. In order to realize this novel flexible device, Thin Film Transistor (TFT) designs are attempted for $50 \mu \mathrm{m}$ Polyimide, flexible substrates, with CTE-12 $\times 10^{-6} \mathrm{PPM} / \mathrm{K}$ and HTE-9 $\times 10^{-6} \%$ RH. TFTs, for ECG designs operate at frequencies $<100 \mathrm{~Hz}$ with sufficient slew rate. Considering the Gate Capacitances Cg, carrier mobilities offered by Indium Gallium Zinc Oxide (a-IGZO) nano crystalline dielectrics and inks, Lov Gate to Source/Drain overlap lengths and requirement of low temperature processing, Thin Film Transistor (TFT) based flexible amplifier design and it's integration with CMOS analog comparator for detection of missing beat, for early warning and printable speaker on Polyamide flexible substrate bendable to a radius of $3.5 \mathrm{~cm}$, computing $W / L$ ratios of every TFT and components of the flexible amplifier, missing pulse(beat) detector and piezo material printing, for speaker are evolved
\end{abstract}

Keywords: TFT, Flexible Biosensor, ECG, Printable Electronics, Body Area Electronics

\section{Introduction}

Sensing Bio-potential signals with flexible epidermal patches is a felt need among clinicians and neuroscientists with integrated circuits capable of amplifying bio-signals in the $\mathrm{mHz}$ to $\mathrm{kHz}$ range, while rejecting large DC offset generated at the electrode-tissue interface. Bio-potentials in general are very week in the range of a few $\mu \mathrm{V}$ to $\mathrm{mV}$, which necessitate high fidelity and low power consumption, in these designs [12]. Bio-potential signals such as Electrocadriogram signals (ECG), Electromyogram (EMG), Electroencephalograph (EEG) to electro-oculograph (EOG) are generated in the human body, in the ranges shown in Table 1.

\begin{tabular}{|l|l|l|}
\hline Medical Signal & Frequency & Amplitude \\
\hline ECG (Electro Cardio Gram) & $0.05-100 \mathrm{~Hz}$ & $5 \mu \mathrm{V}-8 \mathrm{mV}$ \\
\hline EEG (Electro Encephalo Gram ) & $0.5-100 \mathrm{~Hz}$ & $2 \mu \mathrm{V}-200 \mu \mathrm{V}$ \\
\hline Evoke Potential (EP) & $2 \mathrm{Khz}-5 \mathrm{Khz}$ & $20 \mathrm{nV}-20 \mu \mathrm{V}$ \\
\hline EMG (Electro MyoGram) & $0.01 \mathrm{~Hz}-10 \mathrm{Khz}$ & $50 \mu \mathrm{V}-10 \mathrm{mV}$ \\
\hline Electro Occulogram (EOG) & $0.1-10 \mathrm{~Hz}$ & $10 \mu \mathrm{V}-800 \mu \mathrm{V}$ \\
\hline
\end{tabular}

Table 1

Bio-potential signals [12] are generated due to the electrochemical activity of certain class of cells that are components of the nervous, muscular or glandular tissue. Electrically, these cells exhibit a resting potential, and when they are stimulated they generate an action potential. The electrical activity of each cell is described by the ion exchange through the cell membrane. The membrane potential of an inactive cell is called the resting potential. At the rest state, the membrane of the cell is more permeable to $\mathrm{K}+$ than $\mathrm{Na}+$, and $\mathrm{K}+$ concentration of the interior of the cell is much higher than the exterior. Therefore, a diffusion gradient of $\mathrm{K}+$ occurs towards the exterior of the cell making the interior more negative relative to the exterior, which results in an electrical field build up towards the interior of the cell. At steady state, the diffusion gradient of the $\mathrm{K}+$ ions are balanced by the electrical field and the equilibrium is reached with a polarization voltage of nearly $-70 \mathrm{mV}$. 
When the cell is electrically stimulated (through the central nervous system), the permeability of the membrane to $\mathrm{Na}+$ ions increases. Thus, $\mathrm{Na}+$ ions diffuse toward the inside of the cell, resulting in a potential increase of the interior of the cell. As the potential reaches to $+40 \mathrm{mV}$, the permeability of the membrane to Na+ ions decreases and to $\mathrm{K}+$ increases, resulting in a sharp decrease in the membrane potential towards its rest state. This cycle of the cellular potential is called the action potential, and the bio-potential signals, such as EEG, ECG, EMG, are the result of several action potentials produced by a combination of different cells. The origination of action potentials in the heart are depicted in Fig 2 below

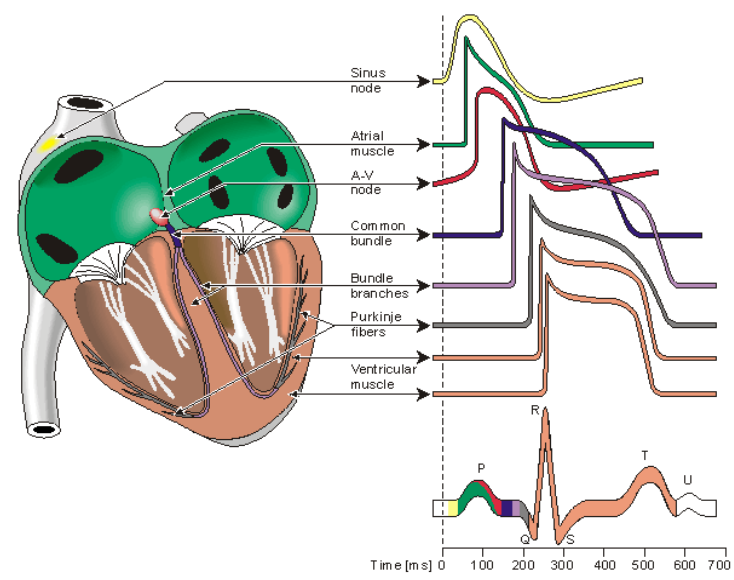

Fig 2

Ideally, bio-potential sensor usually have very large input impedance, a non-zero current should flow from the bio-potential of the body to the input of the sensing block. However, this current is carried by ions in the body, whereas it is carried by electrons on the wires connecting the electrodes to the sensing block. Therefore, a transducer interface is necessary between the body and the sensor block that converts the ionic current into electronic current, or vice versa. This interface is called a bio-potential electrode.

The operation principle of a bio-potential electrode can be described by an electrode-electrolyte interface. In order to allow the current flow between the electrolyte, which has no free electrons, and the electrode, which has no free cations or anions, a chemical reaction has to occur at the interface that can be represented by the following general equations [12]:

$$
\begin{aligned}
& \mathrm{C} \leftrightarrow \mathrm{C}++\mathrm{e}^{-} \\
& \mathrm{A}-\leftrightarrow \mathrm{A}+\mathrm{e}^{-}
\end{aligned}
$$

In this equation, $\mathrm{C}$ and $\mathrm{A}$ stands for the cations and anions in the electrolyte, respectively, and it has been assumed that the electrode is made up of the cations of the electrolyte. Therefore, the cations in the electrode can oxidize at the interface, and the anions coming to the interface can be oxidized to a neutral atom, both resulting in a free electron in the electrode. Thus, current can pass from the electrode to the electrolyte. Therefore, if a metal is inserted in a solution, which has the ions of the same metal and some anions to preserve the neutrality of the solution, the reactions starts to occur depending on the concentration of the cations in the solution. This disturbs the neutrality of the solution, and a charge gradient builds up at the electrode-electrolyte interface, resulting in a potential difference that is called the half-cell potential. The mismatch of the half-cell potential between the reference electrode and the recording electrode is responsible for the differential DC electrode offset voltage.

Bio-potential electrodes can be grouped as, polarisable and non-polarisable electrodes. The perfectly polarisable electrodes have no actual charge transfer between the electrode-electrolyte interface. Thus, such electrodes behave as capacitors and the current is due to the displacement current. On the other hand, the current passes freely across the electrode-electrolyte interface of the non-polarisable electrodes, thus these electrodes behave as a resistor. However, neither of the two types can be fabricated. Thus, practical electrodes are somewhere in between these two types. 


\section{Flexible Non Contact Ecg Sensor}

Various disadvantages of wet $\mathrm{Ag} / \mathrm{AgCl}$ gel electrolyte sensors, in using, for long duration ECG applications have driven researches to evolve dry ECG electrodes. Another important feature required, even in these dry ECG electrodes is the requirement of 24 hours use, including during sleep, without disturbance to the subject or patient, thus epidermal sensors are under evolution. Now, in this work Epidermal Flexible Printable Non Contact Autonomous ECG Sensor Medical Device Patch for Early Warning are evolved.

Flexible biosensor based wearable medical devices can act as excellent terminals in the body area electronics of health monitoring system. Benefiting from the growing advancement of electronics and communication technique are wearable medical devices, which can be worn by people and measure physiological parameters like Electro Cardio Gram (ECG), make it possible to monitor the health status continuously to observe deviations or deterioration, if any, on-line basis, without causing any discomfort to the subjects or patients, in their daily life or routine and could provide warning, even during sleep and wake up the Cardio Vascular or sleep apnea patients, when required. Among all the deaths caused by Cardio Vascular Diseases (CVDs) about two-thirds of them happen in Out-side hospital environment. There is a need to prevent the development of CVDs at its early stage, so as to provide a low cost and efficient way to realize pervasive healthcare.

Cardio Vascular health information involves multiple physiological signals like ECG, BP and SpO2 in multiple modalities. There is a possibility of measuring these parameters, in a completely different way, by wearable medical device from the conventional medical devices without cuffs or hanging electrode wires. Moreover, different wearable medical devices can also take the measurements of the same signal based on different principles, for example Pulse Transit Time (PTT) based BP measurements, using ECG, in cuff less mode. Moreover, epidermal sensing can improve miniaturization, due to the development of new materials. Besides, flexible electronics can make wearable sensors conformal to the skin and even can measure physiological signals unobtrusively. These devices are referred to epidermal electronic systems, which benefit the wearable devices to be thinner, lighter and flexible. In the past Roger et al published on measurements of electrophysiological signals (ECG and EEG), temperature and strain using some of the design transfer methods on to various substrates. Good results have also been reported by Lagally et al and Rogers et. al on the unique electronic, thermo electronic and mechanical properties associated with new class of inorganic flexible semiconductor membrane material systems.

In these ECG Bio-Sensor making, the typical process flow involve, attaching a rigid handler substrate, to a flexible substrate, to facilitate handling in subsequent processing steps, followed by deposition of the thin film device layer via. a solution processing. Inorganic crystalline semiconductor nano materials (free standing sheets with nano meter to sub-micrometer scale thickness) have in the last several years, demonstrated great potential to become a disruptive technology, driven primarily by the successes shown with Group IV crystalline nano materials transferred and tracked on to foreign substrates, including both rigid (eg. Silicon and Glass) and flexible (eg. Plastics and Polymers) substrates.

The technology drivers for this potential are the inherently novel electronic and mechanical properties of these sheets; their flexibility, conformability, biocompatibility and transferability of designs to other hosts; the ability to introduce strain and thus novel properties associated with strain in ways not possible with bulk materials and the ability to integrate membranes of different materials because of the much better bendability of membranes based on PMDS transfer printing processes, originally developed by Rogers et.al and the current nano ink design printing processes and heterogeneous integration of dissimilar materials.

Electroencephalogram (EEG) and Electro Cardio Gram (ECG) sensors require conductive gel to ensure low-impedance electrical contact between the Sensor and Skin making set-up time consuming make the long term recording problematic. In this work, a gel free, non contact EEG/ECG Sensor with on-board electrode that capacitively couples to the skin is evolved. Active shielding of the high impedance input significantly reduces noise pickup and reduces variations in gain as a function of gap distance, between the skin and actual ECG Sensor. The integrated novel medical device sensor combines amplification, missing pulse detection mechanism and peizo electric printed speaker, for warning the CVD or sleep apnea patient, on the flexible patch attached to the skin.

In order to realize this novel device, Thin Film Transistor (TFT) based amplifier designs are attempted for $50 \mu \mathrm{m}$ E Polyimide, flexible substrates, as the sheet material is stable against all chemicals needed for TFT 
fabrication with small Coefficient of Thermal Expansion (CTE) of the order of $12 \times 10^{-6} \mathrm{PPM} /{ }^{0} \mathrm{~K}$ and its small Humidity Expansion Coefficient (HEC) of the order of $9 \times 10^{-6} \% \mathrm{RH}$ [7]. TFTs for ECG amplifier typically need to operate at frequencies $<100 \mathrm{~Hz}$ with sufficient slew rate. Considering the Gate Capacitances $\mathrm{Cg}$, carrier mobilities offered by Indium Gallium Zinc Oxide (a-IGZO) nano crystalline dielectric inks, Lov Gate to Source/Drain overlap lengths and requirement of low temperature processing, the W/L design ratios of every TFT and components of flexible ECG amplifier, missing pulse(beat) detector and peizo electric material area printing are discussed in the publication. Power sources are also printed in such autonomous devices without requirement of an external battery. The process uses a combination of offset printing, inkjet printing, $\mathrm{Al}_{2} \mathrm{O}_{3}$ isolation and contact metallization.

\section{Flexible Integrated Circuit Design}

A gel free non contact [8] ECG sensor design that couples capacitively with skin [8] need to have a front end low noise TFT current mirror based differential amplifier, missing pulse (beat) detector and a printable speaker device at the output and a power source to make it work autonomously as an epidermal device. In the present design exercise undertaken, a printable flexible amplifier and CMOS missing beat detector are designed, to give warning via. a beep signal of a printed speaker, all integrated on a flexible polyamide substrate.

Perhaps the most difficult challenge of ECG sensor is in creating a differential amplifier with very low noise, as signals sensed, reach down to tens of micro volts peak to peak. Ideally, this amplifier need high gain, high common mode rejection ratio (CMRR), high input impedance and high common mode extraction around ECG parameters. It consists of a four stage differential amplifier based on second generation current conveyer (CCII), to meet the application requirements for the gain, linearity and output voltage swing. Current conveyer designs are very good performers, reliable with good enough characteristics for low frequency applications including high input impedance and low power, consists of three terminals A, B and C, as indicated in the Fig. 3

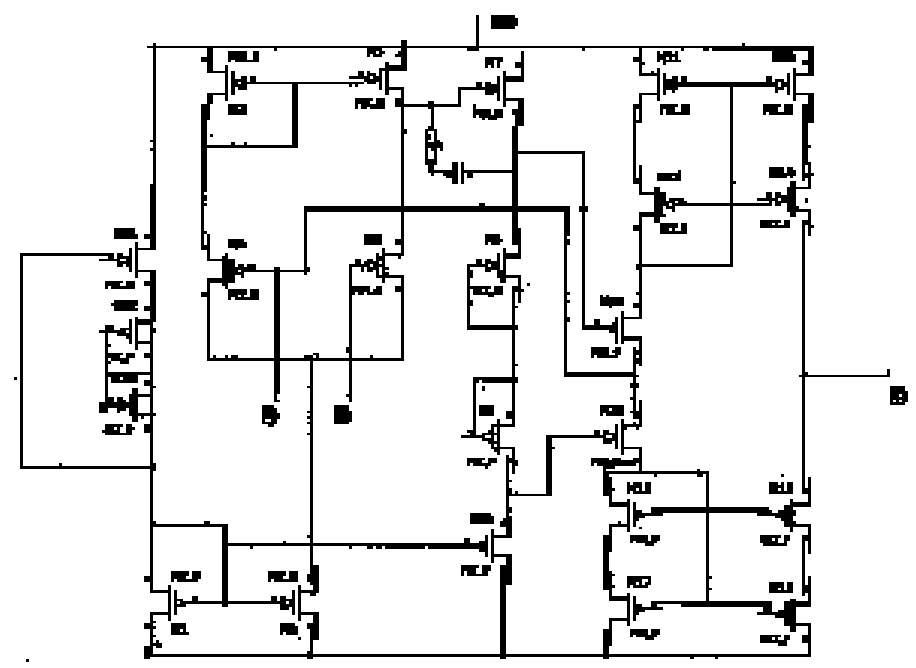

Fig. 3 ( ECG Flexible Amplifier)

\begin{tabular}{|l|l|}
\hline M1, M6,M10,M17,M18 & $1500 / 25 \mu \mathrm{m}$ \\
\hline M2,M3,M19 & $3600 / 25 \mu \mathrm{m}$ \\
\hline M8,M9 & $7200 / 25 \mu \mathrm{m}$ \\
\hline M11,M12 & $750 / 25 \mu \mathrm{m}$ \\
\hline M13,M14 & $1100 / 25 \mu \mathrm{m}$ \\
\hline M15,M16 & $2200 / 25 \mu \mathrm{m}$ \\
\hline M22,M23 & $600 / 25 \mu \mathrm{m}$ \\
\hline M21 & $3000 / 25 \mu \mathrm{m}$ \\
\hline R,C & $1 \mathrm{~K} \Omega, 33 \mathrm{pf}$ \\
\hline
\end{tabular}

Table 2: Designed W/L ratios of Thin Film Transistors, R and C values of Flexible ECG amplifier

Thin Film Transistors (TFTs) M4-M5 form the input differential miller Operational Trans-conductance Amplifier (OTA) stage, ensuring high input impedance levels, required for ECG front end. M2, M3 and M6 transistors are the current sources for the differential pair, and transistor M1 with the Ibias current source are the biasing stage. Bias current can be generated placing a resistor Rbias between M1 transistor and Vdd voltage, 
instead of a current source. Second stage is comprised of transistor M7, a source follower, and diode-connected transistors M8-M9, which ensure the correct biasing for the output stage. Transistor M10 is the current source for that stage, copying the Ibias current from current mirror M1-M10. Finally, transistors M11 to M20 form the output stage. Feedback on M19-M20 transistors ensure a very low A-node parasitic resistance while the cascaded output transistors M12-M14, M16M18 give a very high output C node parasitic resistance, required for missing pulse(beat) detection stages of the ECG Medical device. Transistors M11-M14 and M15-M18 with voltage sources $\mathrm{Vb} 1$ and $\mathrm{Vb} 2$ create two wide swing current mirrors. In the design Rbias for $20 \mu \mathrm{A}$ is created using two Thin Film Transistors (TFTs) M22 and M23. A guaranteed minimum mobility of $7.5 \mathrm{~cm}^{2} / \mathrm{Vs}$ and submicrometer channel lengths are exhibited by a-IGZO TFTs [7]. The capacitor C is an MIM capacitor and printable using a Dielectric Ink such as $\mathrm{HfO}_{2}$. The designed $\mathrm{W} / \mathrm{L}$ ratios of 23 devices used in the flexible printable amplifier of the ECG Medical device patch are indicated in the table 2 above.

TFTs are to be fabricated on flexible $50 \mu \mathrm{m}$ thick Kapton E Polyimide foil. Using a combination of processes such as PECVD for $50 \mathrm{~nm} \mathrm{SiNx}$, Gate contacts with $35 \mathrm{~nm}$ Ti using a liftoff process, 25nm Atomic Layer Deposition(ALD of Al2O3 for gate isolation), between the Source and Drain area a-IGZO nano ink based TFT channel is printed or $15 \mathrm{~nm}$ a-IGZO deposited using RF sputtering at room temperature. To structure the TFT channel in a lift off process, self aligned lithography is used for coating the substrate with evaporated 10 $\mathrm{nm}$ thick $\mathrm{Cr}$ and $60 \mathrm{~nm}$ thick $\mathrm{Au}$ as adhesion layer and top metallization. The maximum process temperature during the fabrication is $150^{\circ} \mathrm{C}$ [7]

\section{Missing Beat Detector}

There has been research on hybrid designs combining Flexible electronics and conventional CMOS designs [4]. In this design a CMOS conventional design indicated in the Fig 4 below based on an analog comparator is integrated with the above ECG Flexible printable amplifier on a flexible polyamide substrate. The design takes ECG at $1 \mathrm{mV}$ levels, at ECG amplifier input and detects whenever ECG beat is missed in a subject and generates a beep in the speaker printed on the same polyamide substrate.

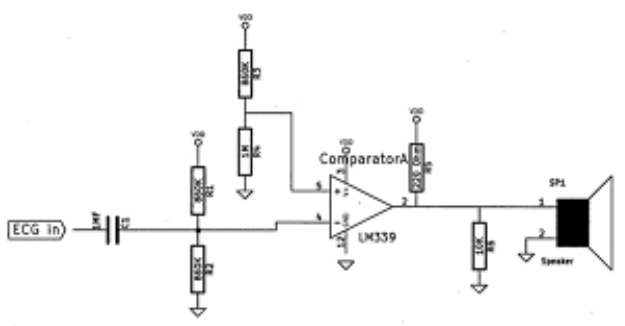

\begin{tabular}{|l|l|}
\hline $\mathrm{C} 1$ & $1 \mu \mathrm{F}$ \\
\hline $\mathrm{R} 1, \mathrm{R} 2, \mathrm{R} 3$ & $860 \mathrm{~K} \Omega$ \\
\hline $\mathrm{R} 4$ & $1 \mathrm{M} \Omega$ \\
\hline R5,R6 & $1 \mathrm{~K} \Omega, 10 \mathrm{~K} \Omega$ \\
\hline Printed Speaker & $5.5 \mathrm{~K} \Omega$ \\
\hline
\end{tabular}

Fig. 4 (Missing Beat Detector)

\section{Printable Speaker and Flexible Power Source}

In order to make the design, flexible and autonomous epidermal patch, a speaker with printed peizo electric layer [5][2] is to be printed on the same polyamide substrate. A speaker of $5.5 \mathrm{~K} \Omega$ impedance in an area of 16 $\mathrm{cm}^{2}$ is sufficient for this ECG sensor patch for audio warning, to indicate missing beat in any subject, to whom the patch is sticked. If the patch has to work autonomously, a flexible power source [15] using Li-O2 material has to be printed with electrodes [15], for powering 14.7 Micro watt flexible device.

\section{ECG Performance Analysis of the Design}

The amplifier has 23 devices, each contributes thermal noise and flicker noise. There is input noise of the order of $7.84 \times 10^{-8} \mathrm{~V} / \sqrt{ } \mathrm{H}$. At the output, with the designed gain and thermal and flicker noise contribution of each of the 23 TFT devices, the maximum noise is not crossing more than $1.57 \mu \mathrm{V} / \sqrt{ } \mathrm{H}$, at $1 \mathrm{Khz}$, as per the 
mathematical computation based design iterations and simulation exercises under taken for the flexible printable amplifier, which get $1 \mathrm{mv}$ ECG signal at its input

$\begin{array}{ll}\text { Power } & 14.7 \mu \mathrm{W} \\ \text { Gain } & 28.57 \mathrm{~dB} \\ \text { Bandwidth } & 100 \mathrm{~Hz} \\ \text { Noise } & 1.57 \mu \mathrm{V} / \sqrt{\mathrm{H}} \\ \text { Common Mode } & \text { Rejection Ratio at } 60 \mathrm{~Hz} \text { is } 57.1 \mathrm{~dB}\end{array}$

\section{Conclusion}

In the current design, only TFT ECG amplifier, Speaker and Battery source are printed. Flexible and printable amplifier and printed speaker are integrated with CMOS analog comparator chips for missing beat detection on the same flexible substrate with a bendability of $3.5 \mathrm{~cm}$ radious. The work is further extendable to print even for TFT based analog comparator in place of CMOS chips used.

\section{Acknowledgement}

Author would like to thank Jodhpur National University, India for allowing the work and support and Centre for Flexible Electronics at IIT Kanpur, whose discussions helped while coordinating with the Centre, as part of his duties.

\section{References}

[1] Bahman Kheradmand-Boroujeni et al., "Analog Characteristics of Fully Printed Flexible Organic Transistors Fabricated With LowCost Mass-Printing Techniques” IEEE Trans. Electron Devices, vol. 51, no. 5, pp 1423-1430, May 2014

[2] A.C. Hubler et al., "Fully mass printed loudspeakers on paper" Organ. Electron., vol. 13, no. 11, pp. 2290-2295, Nov. 2012

[3] S H Kim et al. "Ink-jet printed organic thin-film transistors for low-voltage driven CMOS circuits with solution processed AlOx gate insulator", IEEE Electron Device Lett, vol. 34, no. 2 pp 307-309, Feb 2013

[4] Yingzhe $\mathrm{Hu}$ et al. "Large-Scale Sensing System Combining Large Area Electronics and CMOS ICs for Structural- Health Monitoring”, IEEE Journal of Solid State Circuits, vol. 49, no. 2, Feb 214

[5] R Shabanpour et al. Technische Universitat Dresden, Germany and N Munzenrieder et al. Swiss Federal Institute of Technology, Zurich, Switzerland "A Fully Integrated Audio Amplifier in Flexible a-IGZO TFT Technology for Printed Piezoelectric Loudspeakers", 2015 European Conference on Circuit Theory and Design (ECCTD)

[6] Ana Correia et al. " Design of a Robust General-Purpose Low-Offset Comparator based on IGZO Thin-Film Transistors", IEEE 2015, 978-1-4799-8391-9/15, pp 261-264

[7] Niko Munzenrieder et al. " Flexible Self-Aligned Amorphous InGaZnO Thin-Film Transistors With Submicrometer Channel Length and a Transit Frequency of 135 Mhz", IEEE Trans. Electron Devices, vol 60, no. 9, September 2013

[8] Thomas J. Sullivan, Stephen R and Gert Cauwenberghs University of California, "A Low-Noise, Non-Contact EEG/ECG Sensor" 1-4244-1525-X/07 IEEE 2007

[9] Ye Wang, Wei Sun, Hilmi Volkan Demir, "Influence of Channel Layer Thickness on the Electrical Performances of Inkjet-Printed In-Ga-Zn Oxide Thin-Film Transistors" IEEE Trans Electron Devices, vol. 58, no. 2, February 2011

[10] Koichi Ishida et al., "Large Area Flexible Electronics with Organic Transistors" 978-1-61284-857-0/11 IEEE 2011

[11] Giuseppe Ferri, Vincenzo Stornelli, Alessia Di Simone A CCII-based high impedance input stage for biomedical applications. World Scientific, Journal of Circuits, Systems and Computers 2011; 20 (8): 1441-1447

[12] Joseph D. Bronzino The biomedical engineering handbook, second edition. Springer, IEEE press

[13] D. Jackuline Moni, N. Gopalakrishnan A low power CMOS electrocardiogram amplifier design using 0.18 $\mu \mathrm{m}$ CMOS technology. International Journal of Advancements in Research and Technology 2013; vol. 2 ( issue 2).

[14] Tung-Ming Pan et al., "Structural and Electrical Characteristics of $\mathrm{Yb}_{2} \mathrm{O}_{3}$ and $\mathrm{YbTi}_{\mathrm{x}} \mathrm{O}_{\mathrm{y}}$ Gate Dielectrics for $\alpha$-InGaZnO Thin-Film Transistors" Journal of Display Technology, vol. II, no. 3, March 2015

[15] Qing-Chao Lin et al., "Flexible and Foldable Li-O ${ }_{2}$ Battery based on Paper - Ink Cathode", Advanced Materials 2015, DOI:10.1002/adma.2015.03.0.25 